\title{
Words and Their Crutch
}

\section{Kathryn Sullivan}

the cd changed

with the jolt of a train

switching tracks

lyrics shuffled onto the platform

and stood

hushed

in a city

where silence lets sink

what music propped up

above the metropolis of tangled

rust, a child's balloon punctures

the atmosphere 
Kathryn Sullivan

rising, higher

the smog becomes clouds

rising, higher

becomes a smoky bedroom

where two girls

sitting in hives of blankets, hunting

for words without crutches,

turn to each other with expressions

like crumpled paper 